META-ANALYSIS

\title{
Association of demographic, clinical, laboratory, and radiological characteristics with outcomes of COVID-19 patients: A systematic review and Meta-analysis
}

\author{
Partha Sarathi Biswas ${ }^{1}$, Devosri Sen², Anirban Homchoudhary ${ }^{3}$, Deepika Makkar², Mahima Kapoor ${ }^{1}$, \\ Amandeep Goyal ${ }^{1}$ \\ ${ }^{1}$ Department of Psychiatry, G B Pant Institute of P G Medical Education and Research, Maulana Azad Medical \\ College, New Delhi, India \\ ${ }^{2}$ Private Practitioner, New Delhi, India \\ ${ }^{3}$ Department of Anesthesia, G B Pant Institute of P G Medical Education and Research, Maulana Azad Medical
} College, New Delhi, India

\begin{abstract}
Objectives: The aim of this meta-analysis was to assess association between a composite clinical outcome (severe infection/ required ICU admission/ death) of COVID-19 pneumonia and demographic, clinical, laboratory and radiological findings of these patients.

Methods: A literature search was conducted using the databases PubMed, Medline, Scopus and Web of Science till July. Relative risk (RRs), standardized mean difference (SMDs) and 95\% confidence intervals (Cls) were pooled using random-effects models. We described overall estimates of relevant data of clinical importance from 116,260 COVID-19 pneumonia patients including 19,628 with composite end points from 40 observational studies of 5 countries.
\end{abstract}

Results: The result showed that male gender $(R R=1.24, p<.001)$, older age $(S M D=3.19, P<.001)$ especially $>64$ years $(R R=2.52, P<.001)$ followed aggravated course. Delayed hospitalization $(S M D=.75, p=0.005)$, presence of comorbidity $(R R=1.76, p<.001)$ and multiple co-morbidities $(R R=1.50, p<.001)$ were associated with higher risk of fatal course. Pooled data reported significantly high neutrophil-lymphocyte ratio $(S M D=10.79, p<.001)$, low lymphocyte-toC-reactive protein ratio $(S M D=-3.89, p<.001)$, low platelet count $(S M D=-1.622, p<.001)$, prolonged prothrombin time $(S M D=0.98, p<.001)$, high lactate dehydrogenase $(S M D=6.260, p<.001), D$-dimer $(S M D=1.92, p<.001)$, creatine kinase $(S M D=1.68, p=0.001)$ and interleukin-6 level $(S M D=2.84, P=.001)$ in patients with fatal outcome. Funnel plots and Egger's tests did not reveal any significant publication bias.

Conclusions: Features like older age, male gender, presence of co-morbidities and delayed hospitalization along with the laboratory findings consistent with infection, immune system activation, coagulation disorder and tissue damage could help clinicians to identify COVID-19 patients with poor prognosis at an early stage. J Microbiol Infect Dis 2019; 9(3):121-135.

Keywords: COVID-19, risk factors, predictors, statistical association, outcome, adversity, death/ fatality, laboratory results

\section{INTRODUCTION}

Wuhan, China was the epicenter for outbreak of the coronavirus disease 2019 (COVID-19) and the epidemic then spread all over the world. The predictors of adverse outcome in COVID-19 cases like age, the presence of underlying diseases, secondary infection and elevated blood inflammatory indicators have been reported from individual studies. COVID-19 mortality might be due to virus-activated cytokine storm or fulminant myocarditis [1]. The increased risk of death was also associated median interval from the appearance of initial symptoms to dyspnea in many of the studies [2]. Risk factors for the clinical outcomes of COVID-

Correspondence: Dr. Partha Sarathi Biswas, Department of Psychiatry, G B Pant Institute of P G Medical Education and Research, Maulana Azad Medical College, New Delhi, India Email: drparthas@rocketmail.com

Received: 23 August 2020 Accepted: 31 August 2020

Copyright ( ) JMID / Journal of Microbiology and Infectious Diseases 2020, All rights reserved 
19 pneumonia have been reviewed as of now in only few studies. Parohana et al (2020) [3] published meta analysis on risk factors for mortality in COVID-19 patients from 14 studies with 29,909 COVID-19 infected patients and 1,445 cases of death. There are, until now, four meta-analyses on co morbidities as risk factors for poor outcome of COVID-19 pneumonia [4-7]. Sequential Organ Failure Assessment (SOFA) score have been reported to be high in severe COVID-19 patients [2,8-9]. Research from China $[8,10]$ and US [11] reported low platelet count and coagulation disorder in fatal patients of COVID-19. Elevated serum procalcitonin [12-13] and creatine kinase (CK) [14,2] have also been noticed by some authors. Greater value in neutrophil-lymphocyte ratio (NLR) indicates a patient's overall inflammatory status. Meta analysis on neutrophil-lymphocyte ratio (NLR) and lymphocyte-to-C-reactive protein ratio (LCR) as risk factors is available with pooled data from five studies [15]. But there is no more meta analysis is available on laboratory findings of COVID-19 until now. Chest CT provides a fast, convenient, and effective method to early recognize suspicious cases and might contribute to confine epidemic [16]. Literature is lacking in how bilateral ground glass opacities (one of the most common findings) in CT scan chest can predict prognosis of COVID-19 pneumonia. Thus the deficit in summary description in current literature of risk factors for adverse outcome of COVID-19 patients was motivational for the index study.

Our research question was what demographic, clinical and laboratory or radiological characteristics are associated with the development of severe coronavirus disease 2019 (COVID-19) pneumonia and then progression to fatal morbidity or mortality among these patients? The aim of this meta-analysis was to review all available observational studies on demographic profile, clinical characteristic and laboratory findings of severe or non-severe hospitalized or discharged COVID-19 pneumonia patients, and to assess overall relative risks (RR)/ standardized mean difference (SMD) of age, advanced age, gender, co-morbidities, clinical, hematological and radiological abnormalities in patients with severe/ fatal outcome who required high level of medical care compared to non-severe patients requiring only basic level of medical care.

\section{METHODS}

\section{Databases and Search Strategy}

This study was conducted in accordance with the Meta-analyses Of Observational Studies in Epidemiology (MOOSE) [17] guidelines. We performed a systematic review using the following algorithm search strategy in PubMed, Medline, Scopus and Web of Science: ("coronavirus2019" OR "2019-nCoV" OR "SARSCoV-2" OR "COVID-19") AND ("mortality" OR "death" OR "survival" OR "prognosis" OR "outcome") AND ("co" AND "morbidity" OR "co morbidity") AND ("blood" AND investigation) AND ("laboratory" AND results) AND ("chest" AND "radiography") until July 25, 2020.

\section{Case definition and Outcome variable or end point}

COVID-19 infection was defined as a positive diagnosis based on a real-time fluorescence reverse transcription-polymerase chain reaction (RT-PCR) from a nasopharyngeal swab or gene sequencing of respiratory tract secretions and other samples, or if patients exhibited typical radiological and clinical characteristics of COVID-19 infection despite a negative result from swabbing.

We summarized a set of common events in Figure 1 to determine composite outcome variable. We used composite outcome variable consisted of death, severe pneumonia, SpO2 < $90 \%$, requiring ICU management or use of mechanical ventilator (non-invasive and / or invasive). The study group 'adverse outcome group' (Patients with aggravated/ fatal course requiring higher level of care) was consisted of severely or critically ill patients [according to available guideline in the concerned country, e.g. Diagnosis and Treatment Protocol for Novel Coronavirus Pneumonia, Trial Version 6 [18] or admission to intensive care unit [ICU] or requiring invasive/noninvasive mechanical ventilation (MV) support or in-hospital death. The control group 'stable outcome group' (Patients with favorable/ stable course requiring basic health care facilities) was consisted of mild to moderately severe COVID-19 patients that required either no admission in designated hospital/ ICU/ special ward (i.e., stable course \& discharged without much intervention). This group of patients was treated at home or required only emergency or general hospital admission (Table 1) (Table 1 may be supplied 
by corresponding author if any reader ask for).

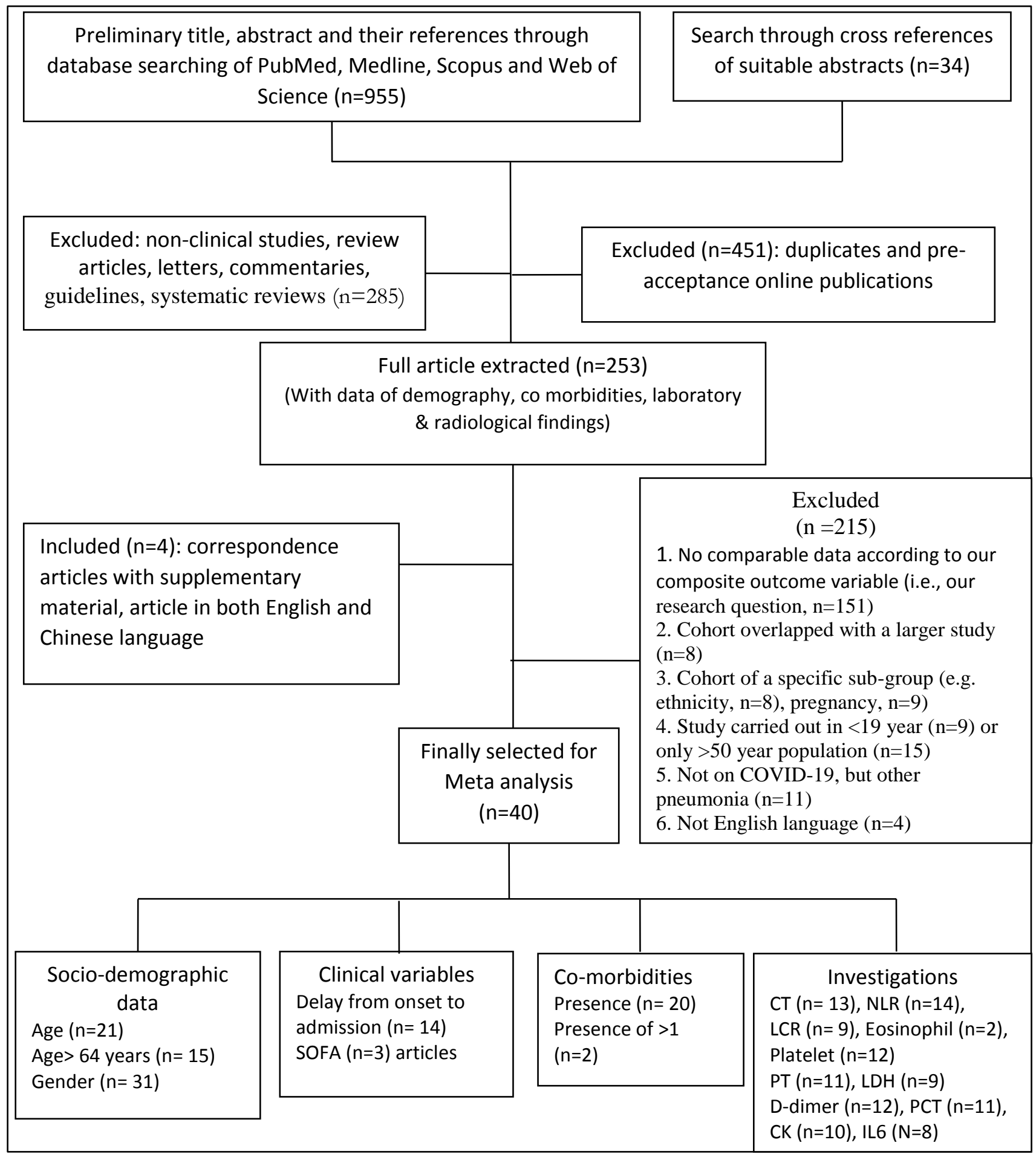

Abbreviation: SOFA, sequential organ failure assessment; CT, computed tomography; NLR, neutrophil-lymphocyte ratio; LCR, lymphocyte-to-C-reactive protein ratio; CK, creatine kinese; PT, prothrobin time; PCT, procalcitonin; IL-6, interleukin 6.

Figure 1. Flow diagram of the number of the studies screened and included into the meta-analyses.

Eligibility Criteria and Study Selection

The inclusion criteria for study selection were- 1) the article must be either accepted or published 
observational study in a peer reviewed journal 2) provided data comparing severely ill in-patients (> 18 yrs. of age) with mild to moderately ill patients etc. 3) diagnosed the COVID-19 pneumonia according to World Health Organization interim guidance or diagnosis and treatment protocol for novel corona virus pneumonia (NCP) (Trial Version 3/4/5) [18], 4) patients having one of the etiological evidences mentioned in case definition mentioned above. The exclusion criteria were- 1) case reports, case series of $<10$ patients, studies that did not report co-morbidities, laboratory or radiological investigations results according to outcome or severity of COVID-19, 2) studies not published in the English language, 3) studies where cohorts were overlapped with each other.

\section{Extraction of variables}

We extracted the following variables: authors' name, periods of data collection, place of data collected, method of diagnosis and severity classification, indications of hospitalization/ ICU admission / putting on MV /discharge, mean age, older patients (>64 years age), gender distribution, blood parameters like absolute count of neutrophil lymphocyte, etc., number of patients in adverse and favorable outcome group. The neutrophil to lymphocyte ratio (NLR) was calculated by dividing absolute neutrophil count by absolute lymphocyte count (taken in each liter). LCR was calculated as follows: lymphocyte count (number/ $\mu \mathrm{L}$ )/C-reactive protein $(\mathrm{mg} / \mathrm{dL})$. Additionally, all $\mathrm{D}$-dimer values were converted to $\mathrm{mg} / \mathrm{L}$ and then entered into dataset.

\section{Statistical analysis}

Means and standard deviations were extrapolated from the median, range, and sample size according to the formula suggested by Hozo et al. [19]. Meta-analysis was performed using STATA 13MP (Stata Corp, College Station, Texas). All Cls were reported with $95 \%$ interval. The variation in $\mathrm{RR}$ or SMD was attributable to heterogeneity when Isquared was more than $50 \%$. The random methods were used for those cases. The estimates were considered significant statistically when overall test statistic (z) was accompanied with $p<0.05$. Subgroups of studies were made depending on country from which the data was collected. Subgroup sensitivity analyses were conducted to explore potential sources of heterogeneity.

\section{RESULTS}

In this study, we have performed a comprehensive exploration of the demographic, clinical, laboratory, and radiological characteristics in 116,260 COVID-19 pneumonia patients from 40 observational studies [1,9,1114,20-53]. They were consisted of 20 single center and 20 multicenter studies of 5 countries [China $(n=30)$, US $(n=5)$, UK $(n=2)$, Italy $(n=2)$, Iran $(n=1)]$, including 19,628 patients with composite end and 96,632 patients without composite end point.

\section{Characteristics of studies}

Thirty-four studies were based on in-patients, whilst six studies used data from infectious diseases registries. Only the sub-sample with information relevant to our outcome variables was extracted from studies with special population [35] or study based on registry data. Sample size ranged from 41 participants up to 44,672 . The proportion of males in studies varied between 46.53 to $75 \%$, with most studies reporting more males than females. Age varied from a median of 25 to 87 years of age, with the oldest median age reported in a study carried out in Italy. All studies were carried out from December 2019 to May 8, 2020.

\section{Risk of Bias and Study Quality}

To assess risk of bias we used the NewcastleOttawa Scale (NOS) [54] and scored in 3 categories (selection, comparability and outcome). A study can be awarded from 0 to 9 score. Any disagreement regarding score for risk of bias was resolved through discussion (Dr. PSB \& Dr. DS). The minimum score was seven in our studies (rated low risk of bias). The results of Beggs's test and Egger's test ( $p r$ and $p$ values respectively: $.592 \& .527$ for age $>64, .708 \&$ .232 for gender.127 \& .037 for presence of co morbidity, and .428 \& .232 for CT scan chest) showed no evidence of publication bias.

\section{Socio-demographic profile and course of illness}

Twenty-one studies, including 19 from China, one each from US and Italy, provided the data for estimating overall effect of age on clinical course of patients with COVID pneumonia. 
Table 1. Descriptions of 40 observational studies participated in the Meta-analysis.

\begin{tabular}{|c|c|c|}
\hline First Author, Place of Study & Type of the study & Primary outcome variables \\
\hline Xiaobo Yang (1), Wuhan & Single-centered, retrospective & Compared between survivor $(n=20)$ and non-survivor $(n=32)$ \\
\hline Dawei Wang (1), Wuhan & Retrospective, single-center & Clinical Characteristics of ICU $(n=36)$ and non-ICU (102) patients \\
\hline X. Wang (2), Fangcang & Single-centered, prospective & Patients with $(n=100)$ or without $(n=912)$ aggravation \\
\hline W. Guan (1), China & Multicentre, retrospective & With $(n=67)$ or without (1032) composite end, and between severe $(n=173) \&$ non-severe $(n=926)$ \\
\hline Jin-jin Zhang (1), Wuhan & Retrospective, single-center & Characteristics of patients with severe $(n=58)$ and non-severe (82) illness \\
\hline Liu et al (3), Hubei & Retrospective, multi-center & Epidemiological Characteristics of mortality $(n=1023)$ and survivor $(n=43649)$ \\
\hline Chaolin Huang, Wuhan & Retrospective, multi-center & Characteristics of ICU $(n=13)$ and non-ICU $(n=28)$ patients \\
\hline Guqin Zhang, Wuhan & Single center, retrospective & Characteristics of severe $(n=166)$ and non-severe $(n=55)$ patients \\
\hline Fei Zhou, Wuhan & Retrospective, multicentre & Characteristics of survivor $(n=137)$ and non-survivor $(n=54)$ patients \\
\hline Chaomin Wu, Wuhan & Retrospective cohort study & Characteristics of patients with $(\mathrm{N}=84)$ and without $(\mathrm{N}=117)$ ARDS, and dead and alive \\
\hline Sijia Tian, Beijing & Retrospective cohort study & Clinical and epidemiological characteristics of severe $(\mathrm{N}=46)$ and common cases $(\mathrm{N}=216)$ \\
\hline Dawei Wang (3), Hubei & Retrospective case series & Clinical course of survivors $(\mathrm{N}=88)$ and non-survivors $(\mathrm{N}=19)$ \\
\hline Tao Chen (1), Wuhan & Retrospective case series & Clinical characteristics and laboratory finding of death $(\mathrm{N}=113)$ and recol \\
\hline Qiurong Ruan, Wuhan & Retrospective multicenter & Clinical predictors of mild \& severe patient outcomes, and those died $(\mathrm{N}=68)$ \& discharged $(\mathrm{N}=82)$ \\
\hline Yan Deng, Wuhan & Retrospective cohort & Clinical characteristics of fatal (109) and recovered cases (116) \\
\hline Geehan Suleyman, US & Retrospective review & Comparative analysis of hospitalized (355) and ambulatory patien \\
\hline Michael G Argenziano, US & Retrospective manual review & Course of patients of emergency department $(n=150)$, inpatient non-ICU $(n=614)$ \& ICU ( $n=236)$ \\
\hline Yichun Cheng, Wuhan & Multicentre, retrospective & Incidence of kidney injury and death $(n=89)$ \\
\hline Christopher M Petrilli, US & Prospective cohort study & Clinical course of patients not admitted (2538) and admitted for critical illness (2741) \\
\hline Annemarie B Docherty, UK & Multicentre prospective & Comparison, between discharged (14968) and died (5165) \\
\hline Davide Colombi, Italy & Retrospective cohort & Discharged (128) and ICU admission or death (108), SARS \\
\hline Lang Wang (4), Wuhan & Retrospective, single-center & Characteristics of survival (274) and death (65) \\
\hline Li Long, Hubei, China & Retrospective & Compared between critical or death $(n=22)$ and discharged patients $(n=156)$ \\
\hline Peng Peng, China & Multicenter, retrospective & Characteristics of death, and composite clinical adverse outcomes $(n=55)$ vs Stable course $(n=648)$ \\
\hline Wei-jie Guan (2), China & Retrospective case & Presence $(n=399) \&$ absence $(n=1191)$ of co-morbidity, and $1(n=269) \&>1$ co-morbidities $(n=130)$ \\
\hline M Nikpouraghdam, Iran & Retrospective & Characterization of survival $(n=2964)$ and non-survival $(n=239)$ \\
\hline Shaobo Shi, Wuhan & Retrospective cohort & Outcomes of patients with $(n=82)$ and without $(n=334)$ cardiac injury were compared. \\
\hline Tricia Tan, London, UK & Prospective cohort & Comparison of characteristic of death (112) and discharged $(n=271)$ patients \\
\hline Kun Wang (6), Wuhan & Multicentre retrospective & Comparison between discharged (307) and dead $(n=33)$ \\
\hline Ai-Ping Yang, Zhejiang & Retrospective & Comparison of NLR, LMR, PLR between severe $(n=24)$ and non-severe $(n=69)$ patients \\
\hline Jingyuan Liu (2), Beijing & Study prospectively & Comparison between mild ( $n=44)$ and severe (moderate to critical) $(n=17)$ patients \\
\hline Safiya Richardson, US & Multi centre case series & Clinical outcomes during hospitalization [discharge alive $(n=2049)$ and dead $(n=553)$ ] \\
\hline Yong Gao, Anhui, China & Retrospective study & Comparison of the hematological parameters between the mild $(n=28)$ and severe (15) groups \\
\hline Kunhua Li, Yuzhong & Retrospective cohort & Compared of ordinary $(n=58)$ COVID-19 cases and severe/critical cases $(n=25)$ \\
\hline Chuan Qin, central China & Retrospective cohort study & Comparison of characteristic between severe $(n=286)$ and non-severe $(n=166)$ patients. \\
\hline Zhongliang Wang (5), China & Descriptive and comparative & Difference of clinical features between the Sp O2 $>=90 \%$ group $(n=55)$ and $<90 \%$ group $(n=14)$ \\
\hline Graziano Onde, Italy & Descriptive and comparative & Comparison between fatalities $(n=1625)$ and survivor $(n=20887)$ \\
\hline Joseph A Lewnard, US & Prospective multi-centre & Comparison of characteristic between died (202) and discharged $(n=893)$ \\
\hline Rong-Hui Du, Wuhan & Prospective single-centre & Compared deceased (21) and survived $(n=158)$ patients \\
\hline Chanyuan Ye, Zhejiang & Retrospective multi-centre & Presence $(n=242)$ and absence $(n=614)$ of co-morbidity, and $1(n=90)$ and $>1$ co-morbidities $(n=152)$ \\
\hline
\end{tabular}


SMD between two limbs of composite outcome variable was significant in all three countries (overall $\mathrm{SMD}=3.19, \mathrm{Cl}=2.17-4.21, \mathrm{Z}=6.12$, $\mathrm{P}<.001$ ) (Figure 2). Nine studies of China, 4 of US, 2 of UK and one each from Iran and Italy showed patients of older than 64 years had an overall higher risk of exacerbation $(R R=2.52$, $\mathrm{Cl}=1.94-3.27, \mathrm{Z}=6.96, \mathrm{P}<.001)$.

Twenty-four studies of China and three of US found that males were at more risk for aggravated course than their female counterpart. Overall males had 1.24 time $(\mathrm{Cl}=1.15-1.34)$ more risk of fatal outcome than female patients $(z=5.51 p=0.000)$.

\section{Clinical profile and course of illness}

Delay in admission after appearance of clinical symptoms had significant effect on outcome $(\mathrm{SMD}=.75, \mathrm{Cl}=.23-1.28 ; \mathrm{z}=2.81 ; \mathrm{p}=0.005)$. An effect of SOFA score on disease outcome was not significant $(S M D=3.933, \quad C l=-3.651$ to 11.518, $z=1.02 p=0.309$ ) when data were pooled from three studies of China (Figure3). Presence of co-morbidities was associated with worse outcome in China $(n=16)$, UK $(n=1)$ and Iran $(n=1)$. The summary effect was significant $(\mathrm{RR}=1.76,95 \% \mathrm{Cl}=1.50-2.07, \mathrm{z}=6.97, \mathrm{p}<.001)$. After pooled data analysis from two studies of China we found that presence of more than one co-morbidity carried 1.5 times more risk of facing fatal outcome $(R R=1.50, C l=1.27-1.77, z=4.80$ $\mathrm{p}<.001)$.

\section{Laboratory findings and course of illness}

The summary results of 11 studies of China, and one each from Italy and US revealed that bilateral chest opacity in CT scan had insignificant effect on outcome $(R R=1.04, C l=$ 1.00-1.08, $z=2.01, p=0.045$ ) (Figure 4). Pooled data from China $(n=13)$ and UK $(n=1)$ reported significantly high values of NLR in patients with fatal outcome $(S M D=10.79, C l=8.89-12.68, z=$ $11.14, p<.001)$. Similarly, overall lower values of LCR from 9 studies of China were associated with aggravated outcome of COVID-19 infection (SMD=-3.89, $\mathrm{Cl}=-4.99$ to $-2.80, \quad \mathrm{z}=6.96$, $\mathrm{p}<.001)$. Eosinophil count had no effect on outcome in a pooled data from two Chinese studies $(\mathrm{SMD}=-.53, \mathrm{Cl}=-1.34$ to $-.28, \mathrm{z}=1.29, \mathrm{p}$ $=0.196)$. Low blood platelet count was reported (11 studies from China and one from Italy) to be significantly associated with adverse clinical course $(\mathrm{SMD}=-1.622, \mathrm{Cl}=-2.64$ to $-.61, \mathrm{z}=3.13 \mathrm{p}$
= 0.002). High PT found in patients (from 11 studies of China) with aggravated clinical course $(\mathrm{SMD}=0.98, \mathrm{Cl}=.07-1.90, \mathrm{z}=2.10, \mathrm{p}=0.036)$. Unfavorable clinical course was also associated significantly with higher values of $\mathrm{LDH}$ (SMD=6.260, I= 4.50- 8.02, $z=6.99 p<.001)$ in COVID-19 patients of 9 countries. Higher Ddimer value lead patients to follow adverse outcome $(\mathrm{SMD}=1.92, \mathrm{Cl}=1.60-2.24, \mathrm{z}=11.76$ $\mathrm{p}<.001)$ when data were pooled from 11 studies of China and one study from UK. Serum PCT had no significant role on outcome (SMD=-.2, $\mathrm{Cl}=-1.48$ to $-1.08, \mathrm{z}=.3, \mathrm{p}=.76$ ) as revealed from 11 studies ( 9 from China and 2 from US). High serum CK from pooled data of 10 studies of China was significantly associated with aggravation of clinical course $(S M D=1.68$, $\mathrm{Cl}=.73-2.64, \mathrm{z}=3.47, \mathrm{p}=0.001$ ). Data of 8 Chinese studies showed significant association of IL-6 with fatal outcome (SMD $=2.84, C l=1.83$ 4.51, $Z=3.36, P=.001$ ). Seven studies (5 from China and 2 from US) revealed that serum ferritin level was not associated with aggravated clinical course $(\mathrm{SDM}=1.26, \mathrm{Cl}=.03-2.5, \mathrm{Z}=2.01$, $\mathrm{P}=.045)$.

\section{DISCUSSION}

Findings from the current systematic review and meta-analysis supported the hypothesis that older age (> 64 years), male gender, presence of co-morbidity, greater number of comorbidities, delayed hospitalization, and some blood parameters (e.g. high NLR, low LCR, high $\mathrm{LDH}, \mathrm{D}$-dimer and $\mathrm{CK}$ ) were associated with higher risk of mortality from COVID-19 infection. Moreover, low platelet count and prolonged PT suggested coagulation defects in COVID-19 patients. Although some observational studies reported significant effect, our meta-analysis did not find any significant association of adverse outcome of COVID-19 pneumonia with high SOFA score, bilateral ground glass opacity in chest CT, low eosinophil count and serum procalcitonin level.

To identify potential sources of heterogeneity, we did subgroup analysis according to the country of presented data. Owing to heterogeneity between studies within a subgroup and between subgroups, randomeffect models were used to estimate the summary effect in our meta-analysis. So it gave us a more conservative estimate of the $95 \% \mathrm{Cl}$. Co-morbidities were determined based on patients' self-report. Co-morbidities were initially 
treated in our analysis as a categorical variable (yes versus no) and subsequently classified based on the number (single versus multiple). It has given an additional edge to our study compared to two other meta-analysis of co morbidities [3, 6]. Experimental studies were excluded because they took either matched sample or parents were randomized across all severities of patients.

The presence of co-morbidities (both cardiometabolic and other) was found to be associated with a higher risk of severe COVID19 and mortality in previous meta analyses [4-5] as well. Earlier meta-analysis by Parohan et al [3] revealed the same result that we have, i.e. older age ( $\geq 65$ years) and male gender were associated with greater risk of death from COVID-19 infection. Older age and chronic diseases are reported to share several features with infectious diseases like pro-inflammatory state, and the attenuation of the innate immune response.

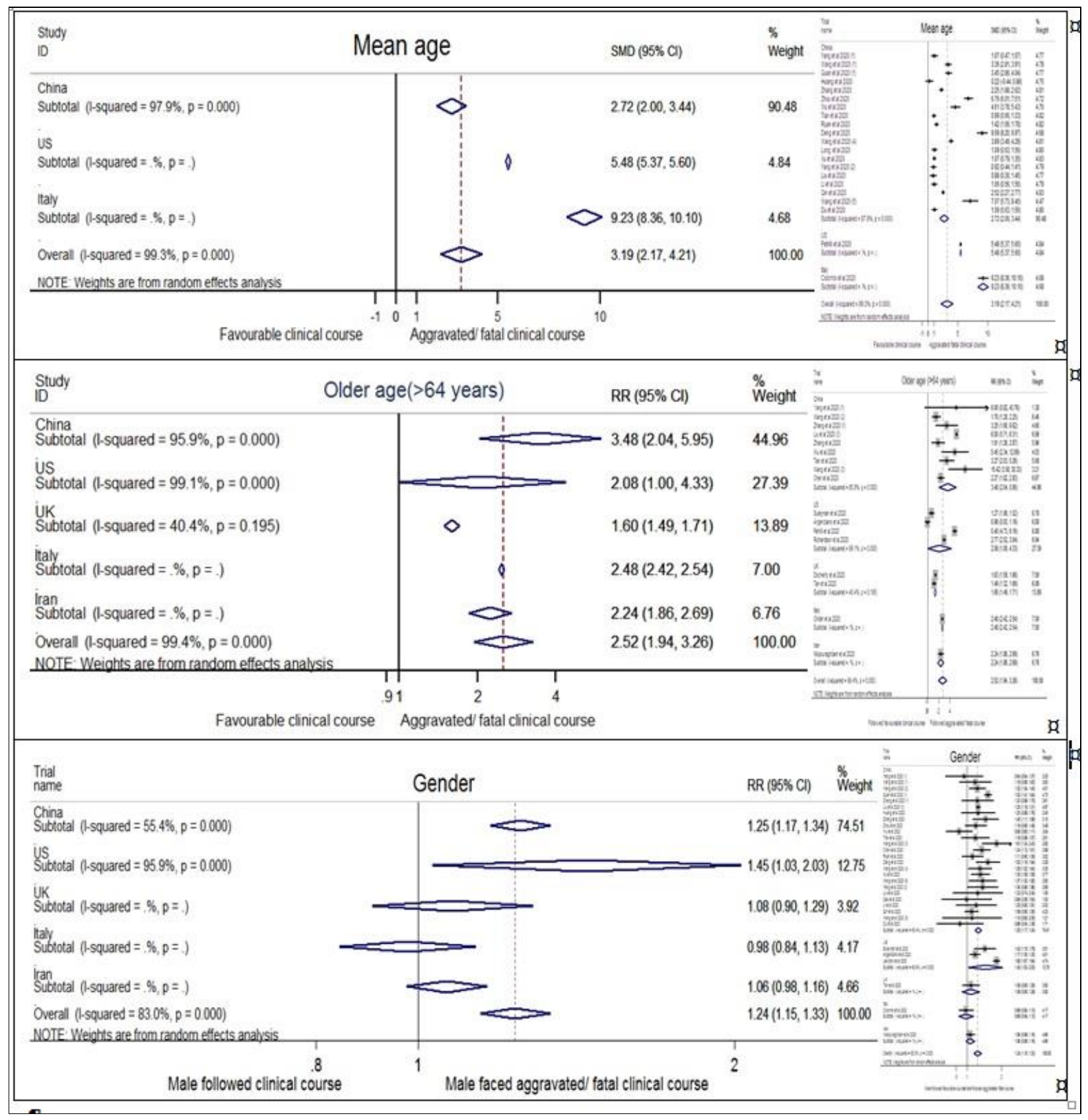

Figure 2. Assosiation between socio-demographic profile and progression of illness. 


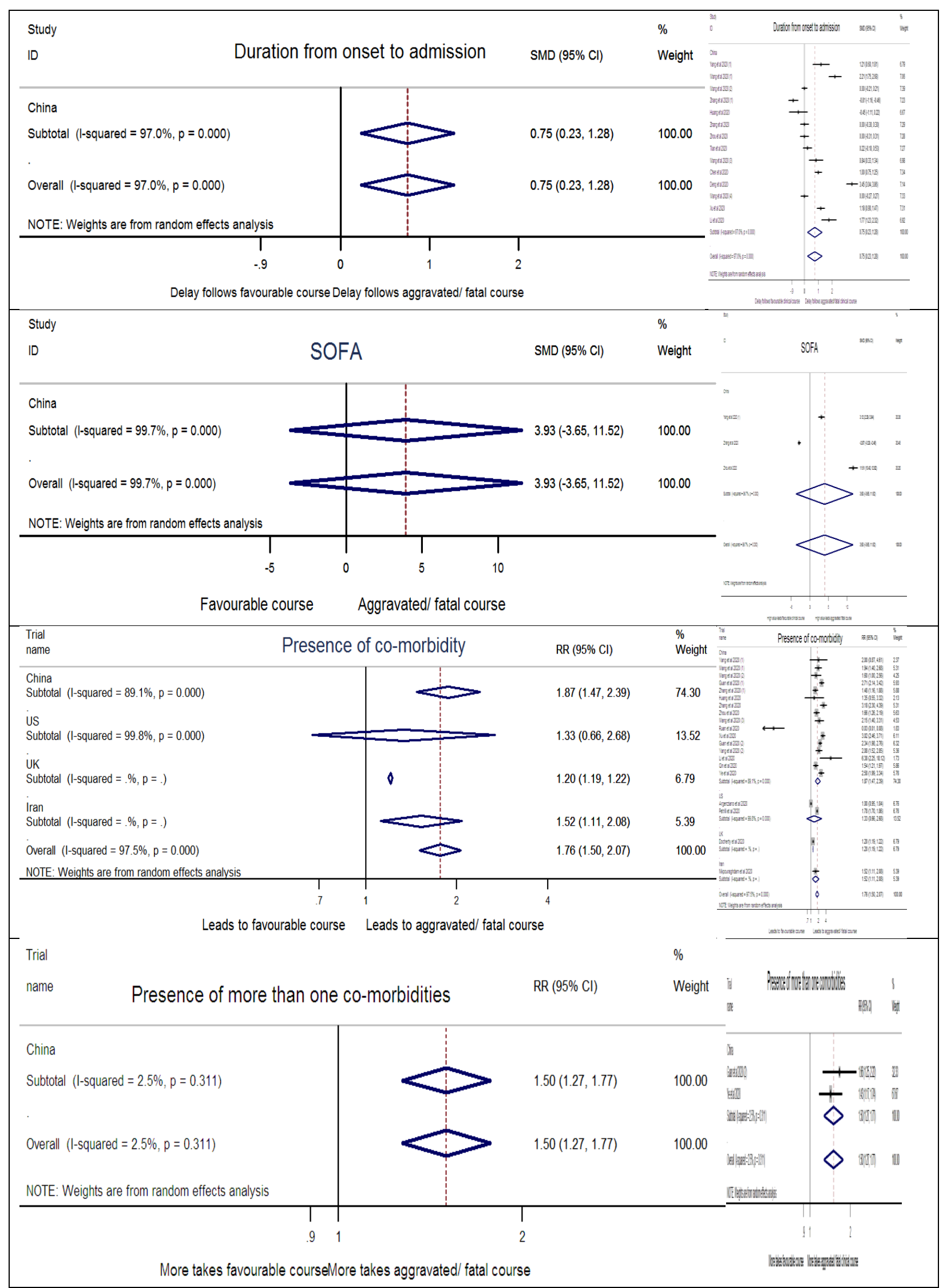

Abbreviation: SOFA, sequential organ failure assessment

Figure 3. Effect of clinical variables on outcome of COVID-19 infection. 


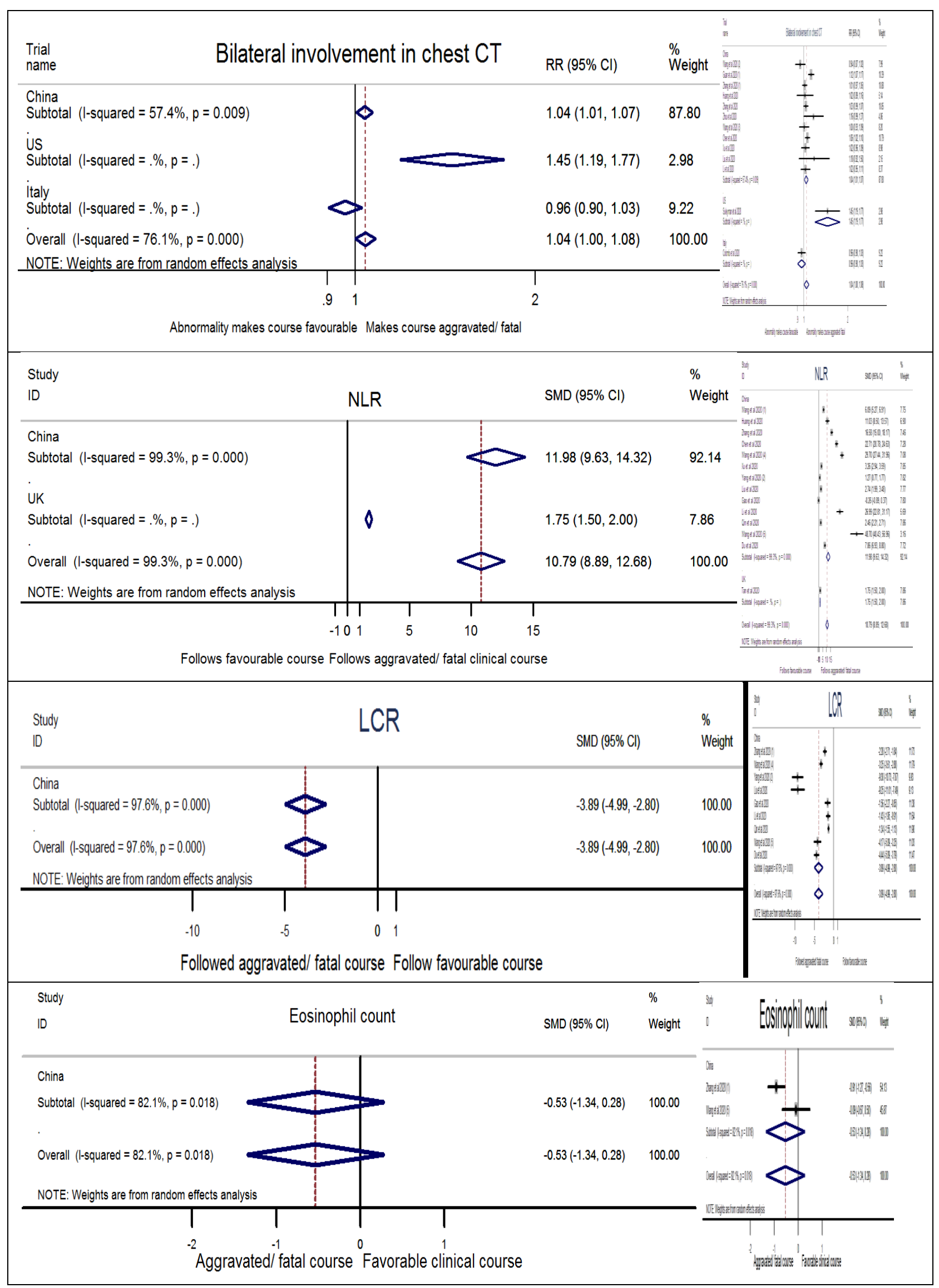




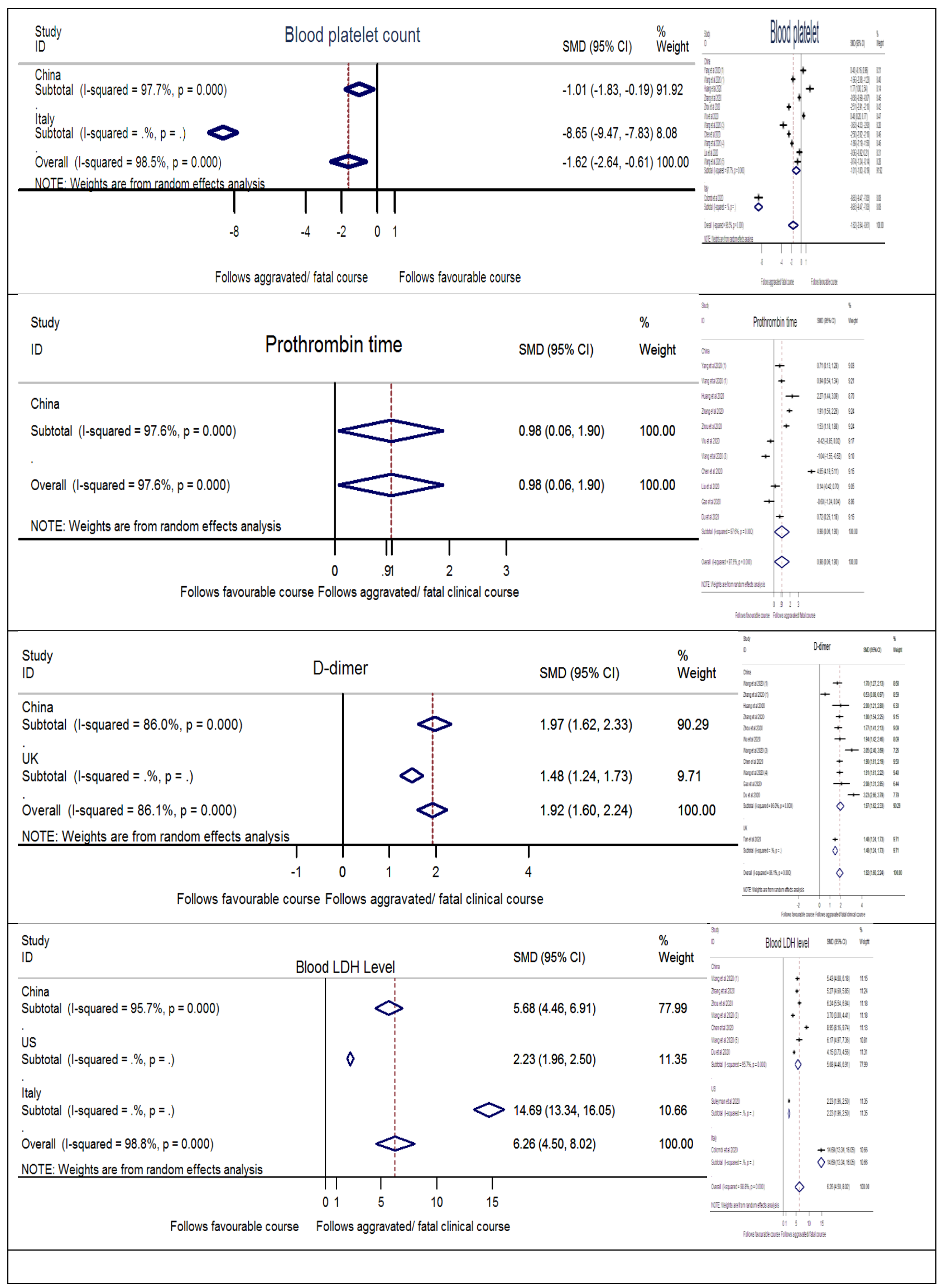




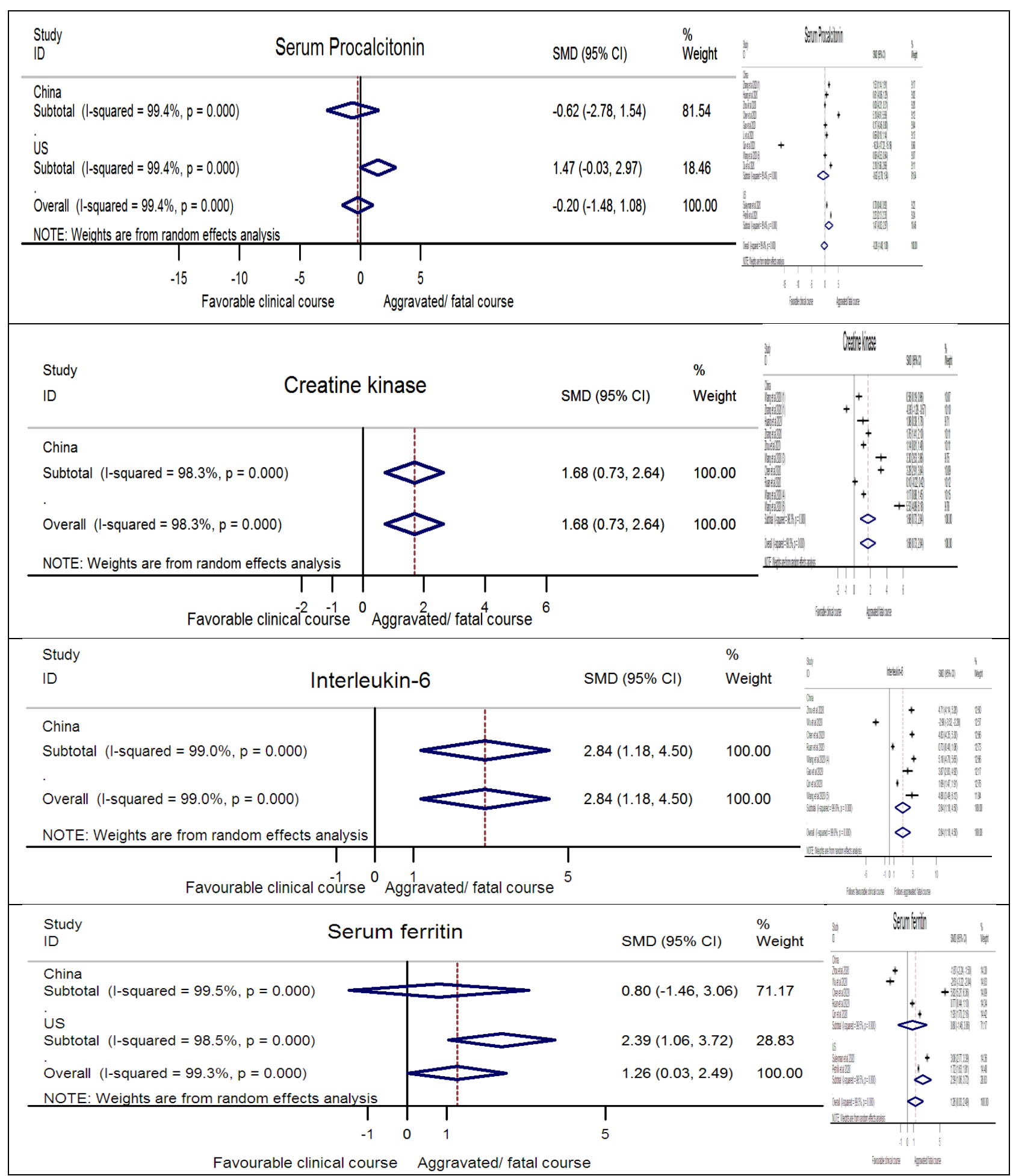

Abbreviation: CT, computed tomography; NLR, neutrophil-lymphocyte ratio; LCR, lymphocyte-to-C-reactive protein ratio; , LDH, lactate dehydrogenase

Figure 4. Laboratory investigations.

Older age has been also been reported as an important risk factor for mortality in SARS and Middle East respiratory syndrome (MERS) [57]. There is evidence of increasing defects in B-cell and T-cell function and the excess production of type 2 cytokines with advanced age. This leads to prolonged pro-inflammatory responses and deficiency in control of viral replication, potentially leading to poor outcome [58]. Jaillon 
et (2019) [59] claimed the role of sex hormones on the responses of adaptive and innate immunity.

In most cohorts $[9,20,29]$, fever was the most common symptom in patients of COVID-19. Thus, the delay of fever manifestation hindered early identification and disease progression. Out of 14 studies of china, 8 showed delay by average 2-8 days led to unfavorable outcome $[9,20,29-31,37,47], 4$ studies revealed no effect on mortality and rest 2 studies showed favorable outcome.

Cytokine storm is thought to play an important role in pathogenesis of COVID-19. Neutrophilia was found in both the lung and peripheral blood of patients with SARS. Same type of lung damage was found with higher numbers of neutrophils and macrophages in the peripheral blood as well as lung tissue in patients with MERS. Neutrophils are the main source of cytokines and chemokines. The generation of cytokine storm can lead to acute respiratory distress syndrome and then death in patients with SARS and MERS [60]. This may explain the positive association between high fever and acute respiratory distress syndrome (ARDS) found in COVID-19 infection [27]. The absolute count of different $T$ cells $(C D 3+T$ cells, CD3+CD8+T cells and CD3+CD4+T) in patients with viral pneumonia was found low in death group than those in the survival group [61]. It signifies that the levels of various inflammatory factors in the non-survival group were higher than those in the survival group (Yang J, 2020) [6]. Although there is report of peripheral lymphopenia [20,22-23], the absolute counts of lymphocytes, were not lower in severe patients compared to non-severe patients probably because of high total numbers of leukocytes in the former [23]. Moreover, increased level of serum C-reactive protein (CRP) has been reported in COVID patients [23]. These observations can explain our findings of NLR and LCR. Xu et al (2020) (1) [16] claimed that higher CT severity score could help clinicians identify patients with potential adverse events. Bilateral chest findings (ground glass appearance) in CT scan did not have overall effect on outcome of COVID-19 pneumonia in our analysis $(R R=1.45, C l=1.19-1.77, z=3.69$, $\mathrm{p}=0.000$ ) (graph 4). In the current literature there is widespread use of CT for the diagnosis of COVID-19 infection. CT scan findings are not pathognomonic as it lacks specificity. Moreover, in about $47 \%$ COVID patients reported to have normal CT scans finding. Thus, some authors like Sun z et al (2020) [62] recommended not using chest CT as a first-line diagnostic or screening tool in COVID-19.

Other abnormal laboratory findings that are reported in literature after infection with SARSCoV-2 are increased level of serum CRP, serum amyloid A (SAA), PCT, prolonged PT, high Ddimer, and $\mathrm{CK}$ which shows sustained inflammatory response and disturbed coagulation mechanism [23]. D-dimer levels greater than $1 \mu \mathrm{g} / \mathrm{mL}$, and higher SOFA score on admission were reported to be associated with higher odds of in-hospital death in some studies [2, 25]. Overall level of D-dimer, in our meta-analysis, was significantly higher in patients with adverse outcome. Infection related marker like IL- 6 and but, in addition, not serum ferritin was found to be related with fatal clinical course. SOFA score is a good diagnostic marker for sepsis and septic shock [63]. But we did not find any effect of SOFA score on clinical outcome from our pooled data of three Chinese studies. Higher leukocyte count and PCT may also be due to secondary bacterial infection.

We found high heterogeneity statistics which was a limitation of this meta-analysis. This may relate to the study designs and large variation among studies in the sample size (41 to 44,672 patients). Additionally, different lengths of followup may be missed the events in some studies leading to heterogeneity as some patients were still in hospital. One of the strengths of this study is that we did subgroup analysis. Secondly, data from China was the sole source for most published meta-analysis so far. But we included studies from 5 countries which improved the validity of our results. Given the limited level of evidence, meta-analysis including more studies from other countries should be conducted in future to find the association between outcome and SOFA score, PCT level, chest radiographic findings with higher power.

\section{Conclusion}

Older age, male gender, presence of comorbidities and delayed hospitalization along with high NLR, low LCR, low platelet count, prolonged PT, and elevated D-dimer, LDH, CK 
levels and IL-6 (suggestive of prominent inflammation infection, immune system activation, coagulation disorder and tissue damage) could help clinicians to identify at an early stage those patients with COVID-19 who have poor prognosis. The older male people with greater number of co morbidities should be included in future SARS-CoV-2 vaccination recommendations.

\section{ACKNOWLEDGMENTS}

We acknowledge Ms Swarnrekha and Dr. Debepriya Sen for their help in manuscript writing and literature search.

\section{Ethical issue}

We took a waiver for the ethical approval from the Institutional Ethical Committee of MAMC, New Delhi.

Declaration of Conflicting Interests: The authors declare that they have no conflict of interest.

\section{Funding:}

No funding received for this work from any of the organizations or so. There is no commercial or proprietary interest in any device, or equipment mentioned in the submitted article. There is no financial interest any author might have (as a consultant, stock owner, employee, evaluator, etc.) in any item mentioned in the article. All authors reported no biomedical financial interests or potential conflicts of interest.

\section{REFERENCES}

1. Ruan Q, Yang E, Wang W, et al. Clinical predictors of mortality due to COVID-19 based on an analysis of data of 150 patients from Wuhan, China. Intensive Care Med 2020; 46(5): 846-848.

2. Zhang G, Hu C, Luo L, et al (2). Clinical features and short-term outcomes of 221 patients with COVID19 in Wuhan, China. Journal of Clinical Virology 2020; 127: 104364

3. Parohana M, Yaghoubib S, Serajic A, et al. Risk factors for mortality in patients with Coronavirus disease 2019 (COVID-19) infection: a systematic review and meta-analysis of observational studies. The Aging Male 2020; 8: 1-9.

4. Wang B, Li R, Lu Z, Huang Y (7). Does comorbidity increase the risk of patients with COVID19: evidence from meta-analysis. Aging 2020; 12(7): 6049-6057.
5. Sing AK, Gillies CL, Sing R, et al. Prevalence of comorbidities and their association with mortality in patients with COVID-19: A Systematic Review and Meta-analysis. Diabetes Obes Metab. 2020 Jun 23;10.1111/dom.14124. doi: 10.1111/dom.14124.

6. Yang J, Zhenga Y, Goua X, et al (3). Prevalence of comorbidities and its effects in patients infected with SARS-CoV-2: a systematic review and metaanalysis. In J Infec Dis 2020; 94: 91-95.

7. Emami A, Javanmardi $F$, Pirbonyeh $N$, et al. Prevalence of Underlying Diseases in Hospitalized Patients with COVID-19: a Systematic Review and Meta-Analysis. Arch Acad Emerg Med 2020; 8(1): e35.

8. Zhou F, Yu T, Du R, et al. Clinical course and risk factors for mortality of adult in-patients with COVID-19 in Wuhan, China: a retrospective cohort study. Lancet 2020; 395: 1054-62.

9. Yang $\mathrm{X}, \mathrm{Yu} Y, \mathrm{Xu}$ J, et al. Clinical course and outcomes of critically ill patients with SARS-CoV-2 pneumonia in Wuhan, China: a single-centered, retrospective, observational study. Lancet Respir Med 2020; 8: 475-81.

10. Wu C, Chen X, Cai $Y$, et al. Risk factors associated with acute respiratory distress syndrome and death in patients with coronavirus disease 2019 pneumonia in Wuhan, China. JAMA Intern Med 2020.

11. Colombi D, Bodini FC, Petrini $M$, et al. Wellaerated Lung on Admitting Chest CT to Predict Adverse Outcome in COVID-19 Pneumonia. Radiology 2020; 296(2):E86-E96.

12. Petrilli CM, Jones SA, Yang J, Rajagopalan $\mathrm{H}$, et al. Factors associated with hospital admission and critical illness among 5279 people with coronavirus disease 2019 in New York City: prospective cohort study. BMJ 2020; 369: m1966.

13. Suleyman G, Fadel RA, Malette KA, et al. Clinical Characteristics and Morbidity Associated With Coronavirus Disease 2019 in a Series of Patients in Metropolitan Detroit. JAMA Network Open 2020; 3(6): e2012270. doi:10.1001/jamanetworkopen. 2020.12270 .

14. Huang C, Wang $Y$, Li X, et al. Clinical features of patients infected with 2019 novel coronavirus in Wuhan, China. Lancet 2020; 395(10223):497-506.

15. Lagunas-Rangel FA. Neutrophil to lymphocyte ratio and lymphocyte to $\mathrm{C}$-reactive protein ratio in patients with severe coronavirus disease 2019 (COVID-19): A meta-analysis. J Med Virol 2020; 127:104364

16. Xu B, Xing $Y$, Peng J, et al (1). Chest CT for detecting COVID-19: a systematic review and metaanalysis of diagnostic accuracy. Eur Radiol 2020; 15;1-8. doi: 10.1007/s00330-020-06934-2.

17. Stroup DF, Berlin JA, Morton SC, et al. Metaanalysis of observational studies in epidemiology: a 
proposal for reporting. Meta-analysis Of Observational Studies in Epidemiology (MOOSE) group. JAMA 2000; 283(15): 2008-2012.

18. National Health Commission of the People's Republic of China. Diagnosis and Treatment Protocol for Novel Coronavirus Pneumonia (Trial Version 6). Available from: http://www.nhc.gov.cn/yzygj/s7653p/202002/8334a83 26dd94d329df351d7da8aefc2.shtml. [Accessed August 2, 2020]

19. Hozo SP, Djulbegovic B, Hozo I. Estimating the mean and variance from the median, range, and the size of a sample. BMC Med Res Methodol 2005; 5(1):13. doi:10.1186/1471-2288-5-13.

20. Wang D, Hu B, Hu C, et al (1). Clinical characteristics of 138 hospitalized patients with 2019 novel coronavirus-infected pneumonia in Wuhan, China. JAMA 2020 7; 323(11): 1061-1069. doi: 10.1001/jama.2020.1585.

21. Wang X, Fang J, Zhu Y, et al (2). Clinical characteristics of non-critically ill patients with novel coronavirus infection (COVID-19) in a Fangcang Hospital. Clin Microbiol Infect 2020; 26(8):1063-1068. doi: 10.1016/j.cmi.2020.03.032.

22. Guan W, Ni Z, Hu Y, et al (1). Clinical Characteristics of Coronavirus Disease 2019 in China. $\mathrm{N}$ Engl J Med 2020; 382:1708-20. doi: 10.1056/NEJMoa2002032

23. Zhang JJ, Dong X, Cao YY, et al (1). Clinical characteristics of 140 patients infected with SARSCoV-2 in Wuhan, China Allergy 2020; 75(7):1730-1741. doi: 10.1111/all.14238.

24. Liu Z, Xing B, Xue ZZ (3). The Epidemiological Characteristics of an Outbreak of 2019 Novel Coronavirus Diseases (COVID-19) in China. Epidemiology Working Group for NCIP Epidemic Response, Chinese Center for Disease Control and Prevention [Abstract in English]. Clin $\mathrm{J}$ Epidemiol 41(2): 145-151. doi:10.3760/cma.j.issn.02546450.2020.02. 003.

25. Zhang G, Hu C, Luo L, et al. Clinical features and short-term outcomes of 221 patients with COVID-19 in Wuhan, China. Journal of Clinical Virology 2020; 127: 104364.

26. Zhou F, Yu T, Du R, et al. Clinical course and risk factors for mortality of adult in-patients with COVID-19 in Wuhan, China: a retrospective cohort study. Lancet 2020; 395: 1054-62

27. Wu C, Chen X, Cai $Y$, et al. Risk Factors Associated with Acute Respiratory Distress Syndrome and Death in Patients With Coronavirus Disease 2019 Pneumonia in Wuhan, China. JAMA Intern Med doi:10.1001/jamainternmed.2020.0994

28. Tian S, Hu N, Lou J, et al. Characteristics of COVID-19 infection in Beijing. Journal of Infection 2020; 80: 401-406.
29. Wang D, Yin Y, Hu C, et al (3). Clinical course and outcome of 107 patients infected with the novel coronavirus, SARS-CoV-2, discharged from two hospitals in Wuhan, China. Critical Care 2020; 24:188.

30. Chen T, Wu D, Chen $\mathrm{H}$, et al. Clinical characteristics of 113 deceased patients with coronavirus disease 2019: retrospective study. BMJ 2020; 368: m1091. doi: 10.1136/bmj.m1091

31. Deng Y, Liu W, Liu K, et al. Clinical characteristics of fatal and recovered cases of coronavirus disease 2019 in Wuhan, China: a retrospective study. Chinese Medical Journal 2020; 133(11)

32. Argenziano MG, Bruce SL, Slater CL, et al. Characterization and clinical course of 1000 patients with coronavirus disease 2019 in New York: retrospective case series. BMJ 2020;369: m1996. doi: 10.1136/bmj.m1996.

33. Cheng $\mathrm{Y}$, Luo $\mathrm{R}$, Wang $\mathrm{K}$, et al. Kidney impairment is associated with in-hospital death of COVID-19 patients. Kidney Int 2020; 97(5):829-838. doi: 10.1016/j.kint.2020.03.005.

34. Docherty $A B$, Harrison EM, Green CA, et al. Features of 20133 UK patients in hospital with covid19 using the ISARIC WHO Clinical Characterisation Protocol: prospective observational cohort study. BMJ 2020; 369: m1985. doi: 10.1136/bmj.m1985.

35. Wang L, He W, Yu X, et al (4). Coronavirus disease 2019 in elderly patients: Characteristics and prognostic factors based on 4-week follow-up. Journal of Infection 2020; 80: 639-645.

36. Long L, Zeng $X$, Zhang $X$, et al. Short-term outcomes of COVID-19 and risk factors for progression. Eur Respir J 2020; 55: 2000990. doi.org/10.1183/13993003.00990-2020.

37. Xu PP, Tian R, Luo $S$, et al. Risk factors for adverse clinical outcomes with COVID-19 in China: a multicenter, retrospective, observational study. Theranostics 2020; 10(14): 6372- 6383. doi: 10.7150/thno.46833

38. Guan W, Liang W, Zhao Y, et al (2). Comorbidity and its impact on 1590 patients with COVID-19 in China: a nationwide analysis. Eur Respir J 2020; 55: 2000547. doi.org/10.1183/13993003.00547-2020.

39. Nikpouraghdam M, Farahani AJ, Alishiri GH, et al. Epidemiological characteristics of coronavirus disease 2019 (COVID-19) patients in IRAN: A single center study. Journal of Clinical Virology 2020;127: 104378.

40. Shi s, Qin m, Shen b, Cai y, Liu t, Yang F, Gong W, Liu $X$ et al. Association of Cardiac Injury with Mortality in Hospitalized Patients With COVID-19 inWuhan, China. JAMA Cardiol doi:10.1001/jamacardio.2020.0950

41. Tan T, Khoo B, Mills EG, et al. Association between high serum total cortisol concentrations and 
mortality from COVID-19. Lancet Diabetes Endocrinol 2020. doi.org/10.1016/S2213-8587(20)30216-3.

42. Wang K, Zuo P, Liu Y, et al (6). Clinical and laboratory predictors of in-hospital mortality in patients with COVID-19: a cohort study in Wuhan, China. Clin Infect Dis 2020; 3(538). doi: 10.1093/cid/ciaa538.

43. Yanga AP, Liub JP, Taoc WQ, et al (2). The diagnostic and predictive role of NLR, d-NLR and PLR in COVID-19 patients. International Immunopharmacology 2020; 84: 106504.

44. Liu Y, Du X, Chen J, et al. Neutrophil-tolymphocyte ratio as an independent risk factor for mortality in hospitalized patients with COVID-19. Journal of Infection. 2020; 81: e6-e12.

45. Richardson S, Hirsch JS, Narasimhan M, et al. Presenting Characteristics, Comorbidities, and Outcomes Among 5700 Patients Hospitalized With COVID-19 in the NewYork City Area. JAMA. doi:10.1001/jama.2020.6775

46. Gao Y, Li T, Han M, et al. Diagnostic utility of clinical laboratory data determinations for patients with the severe COVID-19. J Med Virol 2020;92:c791-796.

47. Li K, Wu J, Wu F, et al. The Clinical and Chest CT Features Associated With Severe and Critical COVID19 Pneumonia. Investigative Radiology 2020; 55(6): 327-331.

48. Qin C, Zhou L, Hu Z, et al. Dysregulation of Immune Response in Patients With Coronavirus 2019 (COVID-19) in Wuhan, China. Clin Infect Dis 2020; 71(15):762-768. doi: 10.1093/cid/ciaa248.

49. Wang Z, Yang B, Li Q, et al (5). Clinical Features of 69 Cases With Coronavirus Disease 2019 in Wuhan, China. Clin Infect Dis. 2020; 71(15):769-777. doi: $10.1093 / \mathrm{cid} / \mathrm{ciaa272}$.

50. Onder G, Rezza G, Brusaferro S. Case-Fatality Rate and Characteristics of Patients Dying in Relation to COVID-19 in Italy. JAMA 2020; 323(18): 1775 1776.

51. Lewnard JA, Liu VX, Jackson ML, et al. Incidence, clinical outcomes, and transmission dynamics of severe coronavirus disease 2019 in California and Washington: prospective cohort study. BMJ 2020; 369: m1923.

52. Du RH, Liang LR, Yang CQ, et al. Predictors of mortality for patients with COVID-19 pneumonia caused by SARSCoV-2: a prospective cohort study. Eur Respir J 2020; 55: 2000524. doi.org/10.1183/13993003.00524-2020.

53. Ye $\mathrm{C}$, Zhang $\mathrm{S}$, Zhang $\mathrm{X}$, et al. Impact of comorbidities on patients with COVID-19: A large retrospective study in Zhejiang, China. J Med Virol 2020; $1-9$

54. Wells G, Shea B, O'Connell D, et al. The Newcastle-Ottawa Scale (NOS) for assessing the quality of non-randomised studies in meta-analyses.
2013,

http://www.ohri.ca/programs/clinical_epidemiology/oxf ord.asp,

55. Dooley KE, Chaisson RE. Tuberculosis and diabetes mellitus: convergence of two epidemics. Lancet Infect Dis 2009; 9: 737-46.

56. Badawi A, Ryoo SG. Prevalence of comorbidities in the Middle East respiratory syndrome coronavirus (MERS-CoV): a systematic review and meta-analysis. Int J Infect Dis 2016; 49:129-33.

57. Hong $\mathrm{KH}$, Choi JP, Hong $\mathrm{SH}$, et al. Predictors of mortality in Middle East respiratory syndrome (MERS). Thorax 2018; 73: 286-289.

58. Opal SM, Girard TD, Ely EW. The immunopathogenesis of sepsis in elderly patients. Clin Infect Dis 2005; 41(7): S504-S512.

59. Jaillon S, Berthenet K, Garlanda C. Sexual dimorphism in innate immunity. Clin Rev Allergy Immunol 2019; 56:308-321.

60. Kim ES, Choe PG, Park WB, et al. Clinical progression and cytokine profiles of Middle East respiratory syndrome coronavirus infection. J Korean Med Sci 2016; 31:1717-1725.

61. Guo L, Wei D, Zhang X, et al. Clinical features predicting mortality risk in patients with viral pneumonia: the MuLBSTA Score. Front Microbiol 2019; 10: 2752.

62. Sun Z, Zhang N, Li Y, Xu X. A systematic review of chest imaging findings in COVID-19. Quant Imaging Med Surg 2020; 10(5):1058-1079. doi.org/10.21037/qims-20-564

63. Ferreira FL, Bota DP, Bross A, et al. Serial evaluation of the SOFA score to predict outcome in critically ill patients. JAMA 2001; 286: 1754-58. 